\title{
Perfil epidemiológico dos atendimentos de um pronto atendimento privado do sul do Brasil
}

Epidemiological profile of care in a private emergency service in southern Brazil

Perfil epidemiológico de la atención en un servicio de emergencia privada en el sur de Brasil

\section{Raíssa Hehn', André Luis Machado Bueno ${ }^{\mathrm{II}}$}

Resumo: Objetivo: analisar o perfil dos atendimentos realizados em um pronto atendimento privado do sul do Brasil. Método: trata-se de um estudo epidemiológico e transversal dos 53.949 registros realizados no ano de 2017, submetidos às técnicas de análise descritiva e inferencial de dados. Resultados: o estudo sugere diferenças geracionais e de gênero capazes de interferir na acessibilidade e na utilização do serviço, bem como no comportamento sazonal dos casos entre os dias da semana. A demanda atendida no serviço não se caracteriza, somente, por situações de urgência e de emergência, fato justificado pela garantia de acolhimento independente da complexidade clínica. Conclusão: predominou atendimentos de menor complexidade, procura ansiosa dos usuários pelo serviço de emergência e a necessidade de incremento em ações que orientem o fluxo dos usuários nas diferentes esferas de atenção.

Descritores: Enfermagem; Perfil de saúde; Serviços médicos de emergência; Necessidades e Demandas de Serviços de Saúde; Instituições privadas de saúde

Abstract: Objective: to analyze the profile of the services provided in a private emergency service in southern Brazil. Method: this is an epidemiological and cross-sectional study of 53,949 records made in 2017, submitted to the techniques of descriptive and inferential data analysis. Results: the study suggests generational and gender differences capable of interfering in the accessibility and use of the service, as well as in the seasonal behavior of cases between the days of the week. The demand met at the service is not characterized only by urgent and emergency situations, a fact justified by the guarantee of reception regardless of clinical complexity. Conclusion: less complex care, anxious demand from users for the emergency service and the need to increase actions that guide the flow of users in different spheres of care predominated.

Descriptors: Nursing; Health Profile; Emergency Medical Services; Health Services Needs and Demand; Health Facilities, Proprietary

Resumen: Objetivo: analizar el perfil de los servicios prestados en un servicio privado de emergencia en el sur de Brasil. Método: este es un estudio epidemiológico y transversal de 53,949 registros realizados en 2017, sometidos a

\footnotetext{
${ }^{\text {I }}$ Enfermeira. E-mail: raissa.hehn@hotmail.com. Taguara, RS, Brasil. ORCID: https://orcid.org/0000-0003-2912-9580

II Enfermeiro. Doutor em enfermagem. Universidade Feevale. Novo Hamburgo, RS, Brasil. E-mail: almachadobueno@gmail.com. ORCID: https://orcid.org/0000-0003-1558-3774
} 
Perfil epidemiológico dos atendimentos de um pronto atendimento privado do sul do Brasil| 2

las técnicas de análisis de datos descriptivos e inferenciales. Resultados: el estudio sugiere diferencias generacionales y de género capaces de interferir en la accesibilidad y el uso del servicio, así como en el comportamiento estacional de los casos entre los días de la semana. La demanda satisfecha en el servicio no se caracteriza solo por situaciones urgentes y de emergencia, un hecho justificado por la garantía de recepción independientemente de la complejidad clínica. Conclusión: predominó la atención menos compleja, la demanda ansiosa de los usuarios por el servicio de emergencia y la necesidad de aumentar las acciones que guían el flujo de usuarios en diferentes ámbitos de atención.

Descriptores: Enfermería; Perfil de Salud; Servicios Médicos de Urgencia; Necesidades y Demandas de Servicios de Salud; Instituciones Privadas de Salud

\section{Introdução}

Os serviços de Pronto Atendimento (PA) são considerados estruturas intermediárias entre as Unidades Básicas de Saúde (UBS), Estratégias de Saúde da Família (ESF) e a rede hospitalar, com funcionamento previsto para as 24 horas do dia, todos os dias da semana. Nesse sentido, eles exigem aparato tecnológico e recursos humanos treinados para assegurar a agilidade e qualidade dos atendimentos. Entende-se que os PAs são fundamentais para a redução da morbimortalidade e sequelas incapacitantes, ocupando papel estratégico na estrutura de assistência à saúde do Sistema Único de Saúde (SUS). ${ }^{1}$

Vários são os fenômenos de caráter urgente e emergente capazes de atingir o indivíduo e as coletividades, a partir de influências culturais, sociais, psicológicas, ambientais e físicas. A ocorrência de traumatismos, processos infecciosos, queimaduras, isquemias, dentre outros agravos à saúde, necessitam de uma estrutura de portas abertas para acolher o paciente e fornecer a assistência necessária. ${ }^{2}$

Segundo a Organização Panamericana de Saúde (OPAS), a cardiopatia isquêmica e o acidente vascular cerebral são os maiores causadores de mortes no mundo, respondendo por um total de 15,2 milhões de óbitos em 2016. Essas doenças têm permanecido como as principais causas de mortalidade global nos últimos 15 anos. $^{3}$ No Brasil, para o ano de 2018, as doenças do 
aparelho circulatório e as neoplasias (tumores), primeiro e segundo lugar em número de óbitos, respectivamente, respondem, aproximadamente, por $44,5 \%$ das mortes. ${ }^{4}$

Agregam-se a essas informações os casos de urgência (situação clínica ou cirúrgica sem risco de morte iminente) e emergência (condições que impliquem sofrimento intenso ou risco iminente de morte) pesquisados em serviços públicos de saúde. Dados destes cenários indicam que as doenças e os agravos de origem neurológica e cardiovascular podem significar mais de $70 \%$ dos serviços prestados. ${ }^{5-6}$

Nesse sentido, traçar o perfil epidemiológico das urgências e das emergências de um serviço privado colabora com os gestores da saúde para a tomada de decisões e intervenções mais eficazes. Cada região tem suas particularidades e necessidades de saúde, solicitando propostas de análise descentralizadas, capazes de possibilitar a transparência dos desafios sociais a serem enfrentados. Assim, essa estratégia possui como principal vantagem fomentar subsídios para a formulação de políticas públicas de saúde sustentáveis e integradas, bem como auxiliar na construção de estratégias de enfrentamento para os problemas de saúde de indivíduos e de coletividades. ${ }^{7}$

Acredita-se, também, que a investigação das características da demanda desses atendimentos pode colaborar na elaboração de critérios para tomada de decisão durante as práticas assistenciais, direcionando esforços para a qualificação de recursos humanos. Enfatizase que o alcance de informações confiáveis, tanto em posição consultiva quanto decisória, é uma estratégica para verificar e melhorar o desempenho de serviços e do sistema de saúde. Mesmo com os avanços contemporâneos relacionados à elaboração das políticas públicas, poucos estudos investigam o uso de evidências e a potencial influência na equidade em saúde. ${ }^{7-8}$

Assim, o objetivo deste estudo é analisar o perfil dos atendimentos realizados em um pronto atendimento privado do sul do Brasil. 
Perfil epidemiológico dos atendimentos de um pronto atendimento privado do sul do Brasil| 4

\section{Método}

Trata-se de um estudo epidemiológico, transversal, submetido e aprovado pelo comitê de Ética e Pesquisa da Universidade Feevale, sob parecer $n^{\circ} 2.973 .903$ de 22 de outubro de 2018. Os dados foram oriundos do banco de registros de um PA privado do estado do Rio Grande do Sul (RS) e correspondem, integralmente, aos 53.949 atendimentos realizados no ano de 2017.

A região estudada é composta por 10 municípios, com área aproximada de $1.732,8 \mathrm{Km}^{2}$, 219.969 habitantes, 73,03 anos de expectativa de vida ao nascer e taxa de analfabetismo de $4,31 \%$ para pessoas de 15 anos ou mais. ${ }^{9} \mathrm{O}$ PA é referência em urgência e emergência para todas as faixas etárias na região. Conta com serviços de laboratório, exames radiológicos, ultrassonografia, tomografia, ressonância magnética, endoscopia e colonoscopia. É composto, estruturalmente, no que se refere aos espaços assistenciais, pela recepção, sala de espera, sala de emergência e de classificação de risco, posto de enfermagem, cinco consultórios, sala de curativos, sala de sutura, sala de gesso, sala de observação pediátrica e observação adulta. O serviço utiliza para triagem dos usuários o protocolo de Acolhimento e Classificação de Risco da Política Nacional de Humanização (HumanizaSus). ${ }^{10}$

As variáveis investigadas foram: sexo, raça, faixa etária, mês, dia da semana, turno de atendimento, classificação de risco, tempo de permanência, queixa principal, desfecho e exames realizados. O acesso aos registros dos prontuários eletrônicos contou com o auxílio de um profissional do setor de Tecnologia da Informação (TI), vinculado à instituição coparticipante, com dados disponibilizados em uma planilha eletrônica.

As variáveis nominais foram expressas em análises de frequência e as variáveis contínuas por meio de média e desvio padrão. Para verificar as possíveis associações entre a classificação de risco na hora do atendimento e as variáveis relacionadas ao perfil dos usuários analisados, foi utilizado o teste qui-quadrado $\left(\chi^{2}\right)$, de acordo com as suposições do teste de normalidade (teste 
de Kolmogorov-Smirnov) e intervalo de confiança de 95\%. Para análise dos dados, foi utilizado o programa SPSS 21.0.

\section{Resultados}

Os resultados dizem respeito aos 53.949 atendimentos realizados no período do estudo, contidos e influenciados pelo recorte espaço-temporal e pelo processo histórico-cultural do local de inserção do serviço de saúde. Dessa forma, a Tabela 1 apresenta as características sociodemográficas dos usuários atendidos em relação ao sexo, raça e faixa etária.

Tabela 1 - Sexo, raça e faixa etária dos usuários atendidos em um Pronto Atendimento privado, do Sul do Brasil, para o ano de 2017. ( $\mathrm{N}=53.949)$

\begin{tabular}{lrr}
\hline \multicolumn{1}{c}{ Variáveis } & $\mathbf{N}$ & $\mathbf{\%}$ \\
\hline Sexo & & \\
Feminino & 33.918 & 62,9 \\
Masculino & 20.006 & 37,0 \\
Não informado & 25 & 0,1 \\
Raça & & \\
Sem informação & 53.883 & 99,8 \\
Branco & 46 & 0,1 \\
Pardo & 20 & $-^{*}$ \\
Faixa etária & & \\
Menos de um ano & 3 & $-*$ \\
1 a 4 anos & 1914 & 3,5 \\
5 a 9 anos & 1670 & 3,1 \\
10 a 14 anos & 1328 & 2,5 \\
15 a 19 anos & 2125 & 3,9 \\
20 a 29 anos & 10745 & 19,9 \\
30 a 39 anos & 12876 & 23,9 \\
40 a 49 anos & 9159 & 17,0 \\
50 a 59 anos & 6583 & 12,2 \\
60 a 69 anos & 3589 & 6,7 \\
70 a 79 anos & 2123 & 3,9 \\
\hline 0 anos e mais & 1834 & 3,4 \\
\hline
\end{tabular}

Fonte: banco de dados da instituição co-participante da pesquisa. * Percentuais menor que $0,05 \%$. 
Perfil epidemiológico dos atendimentos de um pronto atendimento privado do sul do Brasil| 6

As mulheres foram responsáveis pela maioria dos atendimentos realizados no período investigado. Em relação aos registros de raça, observou-se uma taxa de 99,9\% de não preenchimento dessa variável. A faixa etária de predomínio dos atendimentos diz respeito aos indivíduos de 20 a 49 anos, com 60,8\% dos registros. A Tabela 2 apresenta a procura de assistência de acordo com o mês, dia da semana, turno de atendimento, classificação de risco estabelecida e tempo de permanência na instituição.

Tabela 2 - Características dos atendimentos realizados em um Pronto Atendimento privado, do Sul do Brasil, para o ano de 2017. $(\mathrm{N}=53.949)$

\begin{tabular}{|c|c|c|}
\hline Variáveis & $\mathbf{N}$ & $\%$ \\
\hline \multicolumn{3}{|l|}{ Mês de atendimento } \\
\hline Janeiro & 4.164 & 7,7 \\
\hline Fevereiro & 3.867 & 7,1 \\
\hline Março & 4.687 & 8,7 \\
\hline Abril & 4.148 & 7,7 \\
\hline Maio & 4.633 & 8,6 \\
\hline Junho & 4.672 & 8,6 \\
\hline Julho & 4.194 & 7,8 \\
\hline Agosto & 4.669 & 8,6 \\
\hline Setembro & 4.409 & 8,2 \\
\hline Outubro & 5.055 & 9,4 \\
\hline Novembro & 4.650 & 8,6 \\
\hline Dezembro & 4.801 & 8,9 \\
\hline \multicolumn{3}{|l|}{ Dia da semana } \\
\hline Domingo & 5.872 & 10,9 \\
\hline Segunda-feira & 9.395 & 17,4 \\
\hline Terça-feira & 9.770 & 18,1 \\
\hline Quarta-feira & 6.983 & 13,0 \\
\hline Quinta-feira & 7.629 & 14,1 \\
\hline Sexta-feira & 7.414 & 13,7 \\
\hline Sábado & 6.886 & 12,8 \\
\hline \multicolumn{3}{|l|}{ Turno de atendimento } \\
\hline Das 00:00 às 07:00 & 2694 & 5,0 \\
\hline Das 07:00 às 13:00 & 22.170 & 41,1 \\
\hline Das 13:00 às 19:00 & 18.357 & 34,0 \\
\hline Das 19:00 às 00:00 & 10.728 & 19,9 \\
\hline \multicolumn{3}{|c|}{ Classificação de risco segundo o protocolo Humaniza SUS } \\
\hline Verde * & 36.845 & 68,3 \\
\hline Amarelo $^{+}$ & 14.291 & 26,5 \\
\hline
\end{tabular}




\begin{tabular}{lrr} 
Vermelho ${ }^{*}$ & 2.052 & 3,8 \\
Azul § & 761 & 1,4 \\
Tempo de permanência & 47.981 & 88,9 \\
Alta no mesmo dia & 5.507 & 10,3 \\
Um dia & 392 & 0,7 \\
Dois dias & 69 & 0,1 \\
Sem Informações & & \\
\hline Fonte: banco de dados da instituição co-participante da pesquisa. & \\
${ }^{*}$ engloba quadros clínicos pouco urgentes, com atendimento em até 120 minutos \\
${ }^{\dagger}$ engloba quadros clínicos de urgência, com atendimento em até 60 minutos \\
${ }^{*}$ engloba quadros clínicos de emergência, requer atendimento imediato \\
§ engloba quadros clínicos não urgentes, com atendimento em até 240 minutos
\end{tabular}

O mês de outubro foi o mais procurado pela população, com 9,4\% dos atendimentos. Os dias com maior demanda foram nas terças-feiras $(18,1 \%)$ e às segundas-feiras $(17,4 \%)$. O turno com maior fluxo de usuários foi o da manhã (07:00 às $13: 00)$, com $41,1 \%$ dos atendimentos. A maioria dos casos foram classificados como verde $(68,3 \%)$ e, no que se refere ao tempo de permanência na unidade, $89 \%$ dos casos não chegaram a um dia. A Tabela 3 apresenta as principais queixas dos usuários (categorizadas de acordo com o sistema afetado) ao acessarem o serviço. As queixas gastrointestinais e musculoesqueléticas perfizeram $55,2 \%$ dos atendimentos.

Tabela 3 - Motivo dos atendimentos em um Pronto Atendimento privado, do Sul do Brasil, para o ano de 2017. $(\mathrm{N}=53.949)$

\begin{tabular}{|c|c|c|}
\hline Queixas & $\mathbf{N}$ & $\%$ \\
\hline Gastrointestinais (diarreia, vômitos, dor abdominal, outras). & 19.666 & 36,4 \\
\hline $\begin{array}{l}\text { Musculoesqueléticas (dor lombar, trauma por quedas e acidentes de } \\
\text { trânsito, outras). }\end{array}$ & 10.132 & 18,8 \\
\hline $\begin{array}{l}\text { Neurológicas (cefaleia, rebaixamento de sensório, parestesias, } \\
\text { convulsões, outras). }\end{array}$ & 7.391 & 13,7 \\
\hline Respiratórias (tosse, febre, dor de garganta, outras). & 6.832 & 12,7 \\
\hline Excretórias (disúria, hematúria polaciúria, outras). & 3.559 & 6,6 \\
\hline Cardiovasculares (hipertensão, arritmia, dor torácica, outras). & 2.669 & 4,9 \\
\hline Tegumentares (ferimentos corto contuso, lesões cutâneas, outras). & 1.505 & 2,8 \\
\hline $\begin{array}{l}\text { Psiquiátricas (ansiedade, crise de pânico, tentativa de suicídio, } \\
\text { outras). }\end{array}$ & 686 & 1,3 \\
\hline $\begin{array}{l}\text { Ginecológicas/obstétricas (sangramento vaginal, perda de líquido na } \\
\text { gestação, corrimento vaginal, outras). }\end{array}$ & 431 & 0,8 \\
\hline Outros $^{*}$ & 326 & 0,6 \\
\hline
\end{tabular}


Perfil epidemiológico dos atendimentos de um pronto atendimento privado do sul do Brasil| 8

Oftalmológicas (traumas oculares, hiposfagma, prurido, outras).

$274 \quad 0,5$

Auditivas (otalgia, otorragia, outras).

$256 \quad 0,5$

Urológicas (trauma testicular, secreção peniana, outras).

$122 \quad 0,2$

Endócrinas (hipoglicemia, hiperglicemia).

100

0,2

Fonte: banco de dados da instituição co-participante da pesquisa.

Outros*: não identificado descrição no prontuário.

A Tabela 4 apresenta o desfecho dos atendimentos, com 93,6\% dos usuários recebendo alta após serem atendidos. No que se refere aos exames solicitados, os laboratoriais e os de raio x perfizeram $88,4 \%$ do total de solicitações no período.

Tabela 4 - Desfecho dos atendimentos e exames realizados em um Pronto Atendimento privado, do Sul do Brasil, para o ano de 2017. ( $\mathrm{N}=53.949)$

\begin{tabular}{|c|c|c|}
\hline Variável & $\mathbf{N}$ & $\%$ \\
\hline \multicolumn{3}{|l|}{ Desfecho } \\
\hline Alta * & 50.526 & 93,6 \\
\hline Internação hospitalar & 1.770 & 3,3 \\
\hline Encaminhado ao especialista & 1.175 & 2,2 \\
\hline Desistência $^{+}$ & 207 & 0,4 \\
\hline $\begin{array}{l}\text { Retorno para reavaliação após } \\
\text { tratamento }\end{array}$ & 153 & 0,3 \\
\hline Evadiu-se/alta a pedido ${ }^{*}$ & 117 & 0,2 \\
\hline Óbito & 1 & $-\S$ \\
\hline \multicolumn{3}{|l|}{ Exames $(\mathrm{N}=12.312)$} \\
\hline Laboratorial & 6.976 & 56,7 \\
\hline Raio X & 3.894 & 31,7 \\
\hline Ultrassom & 876 & 7,1 \\
\hline Tomografia computadorizada & 537 & 4,3 \\
\hline Ultrassonografia com Doppler & 26 & 0,2 \\
\hline Angiotomografia & 3 & $-\S$ \\
\hline
\end{tabular}

Fonte: banco de dados da instituição co-participante da pesquisa.

* Alta: após avaliação médica.

${ }^{+}$Desistência: após classificação de risco, paciente não aguarda consulta médica.

${ }^{\ddagger}$ Evadiu-se/alta a pedido: paciente recusa-se a receber o tratamento prestado.

$\S$ Percentuais menores que $0,05 \%$.

A Tabela 5 relaciona a classificação de risco com as características dos atendimentos e o perfil sociodemográfico dos usuários. Houve associações estatisticamente significativas para os dias da semana, tempo de permanência, sexo e faixa etária. 
Tabela 5 - Associações entre a classificação de risco com as características dos atendimentos e o perfil dos usuários atendidos em um Pronto Atendimento no Sul do Brasil, para o ano de 2017.

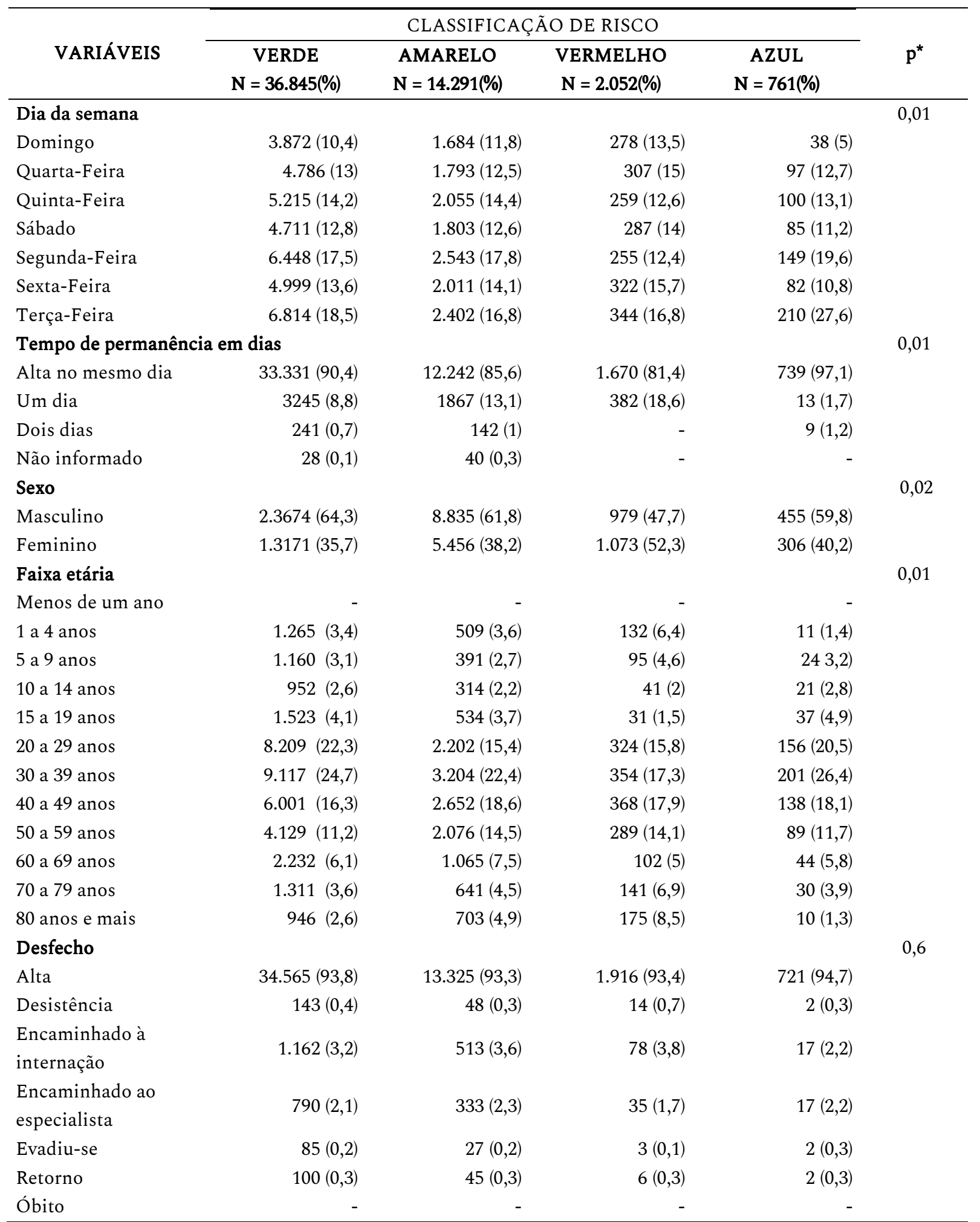

Fonte: banco de dados da instituição co-participante da pesquisa.

*teste qui-quadrado $\left(\chi^{2}\right)$ com intervalo de confiança de $95 \%$. 
Perfil epidemiológico dos atendimentos de um pronto atendimento privado do sul do Brasil| 10

\section{Discussão}

A procura por atendimento nos serviços de saúde pelo sexo masculino é inferior à feminina, e esse comportamento pode encontrar explicações na maior expectativa de vida das mulheres. No entanto, com base nos resultados apresentados, acredita-se que os homens, culturalmente, são vistos como sujeitos invulneráveis, fortes e viris. Muitos acreditam que, ao procurarem por atendimento, serão vistos como fracos, temerosos e inseguros. Outro fator agravante deste cenário é o não exercício de medidas preventivas, de autocuidado, de forma rotineira. Medidas estas amplamente praticadas pelo sexo feminino. Estes, entre outros fatores, contribuem para o agravo da saúde masculina, constituindo-se em um grave problema de saúde pública. ${ }^{11}$

A associação da classificação de risco com a variável sexo indica que os homens procuram atendimento quando seu estado clínico já se agravou. Acredita-se que essa situação se deve ao modelo hegemônico de masculinidade, uma vez que heterossexualidade, agressividade, ser forte e imbatível, compõem os requisitos para ser um homem ideal. A baixa adesão masculina às medidas de autocuidado justifica, em parte, a gravidade dos casos e contribui para os elevados números de morbidade entre os homens. ${ }^{11-12}$

Em um estudo ${ }^{12}$ relacionado aos motivos de mulheres para procurarem assistência em PA, foram citados como fatores influenciadores dessa atitude a falta de resolutividade e acolhimento da atenção primária e as dificuldades de agendamentos de consultas gerais e com especialistas. Nesse sentido, entende-se que a busca por unidades de urgência e emergência justifica-se pela maior capacidade diagnóstica (exames de imagem e laboratoriais) e resolutividade dos casos. Outro fator contribuinte para esta situação é o acúmulo de funções cotidianas, o que pode colaborar para o aumento da procura de atendimento feminino fora do seu horário de trabalho. ${ }^{12}$ 
Em relação aos registros da variável raça, observou-se uma elevada taxa de subnotificação desse dado. Neste segmento, constata-se a necessidade de aprimoramento na execução do registro de atendimento e a impossibilidade de utilização dessa variável na proposta analítica.

Nesse sentido, dados sobre a percepção dos profissionais de saúde (gestores e trabalhadores assistenciais) sobre as causas de subnotificação em serviços públicos apresentam características multifacetadas. Entre essas diferentes causas, é possível destacar: problemas no diagnóstico do paciente; complexidades das doenças ou agravos; rotinas e protocolos dos serviços; capacidade técnica dos recursos humanos; não valorização da Vigilância Epidemiológica, dentre outras. Cabe ressaltar a importância de contextualização dos diferentes espaços de trabalho dos profissionais de saúde que, substancialmente, interferem nas práticas assistenciais desenvolvidas, entre elas, a qualidade do registro. ${ }^{13}$

Atendimentos destinados a adultos foram os mais prevalecentes na faixa etária entre os 20 e 49 anos, e significantes, estatisticamente, entre os indivíduos de 30 a 39 anos. O processo de urbanização, a intensa migração, múltiplas tarefas sem intervalo para descanso, alimentação inadequada, sedentarismo, entre outros, expõem a população a fatores de risco que contribuem para o surgimento e cronicidade das doenças. Soma-se a essas causas o aumento dos transtornos depressivos e quadros de morbimortalidade devido às doenças crônicas não transmissíveis, fomentam comportamentos e práticas de saúde nocivas adotados nos cotidianos de vida. ${ }^{8}$

No que se refere aos casos de violência, todo ano, mais de um milhão de pessoas perdem suas vidas e muitas outras sofrem lesões não fatais, resultantes de eventos autoinfligidos, interpessoais ou coletivos. Agravos oriundos de atos violentos estão entre as principais causas de morte de pessoas na faixa etária de 15 a 44 anos no mundo. Seu custo se traduz em bilhões gastos, anualmente, com assistência à saúde, dias de trabalho perdidos, incapacidades laborais e sofrimento de vítimas e familiares. ${ }^{14}$ 
Perfil epidemiológico dos atendimentos de um pronto atendimento privado do sul do Brasil| 12

Agregam-se a esses dados os acidentes de trânsito que interrompem a vida de, aproximadamente, 1,35 milhão de pessoas no mundo. Somam-se a esse número, 20 a 50 milhões de indivíduos que sofrem lesões não fatais, muitas delas resultando em incapacidade. O custo financeiro dos acidentes de trânsito é de, aproximadamente, $3 \%$ do produto interno bruto (PIB) de um país. Mais da metade das mortes são de vulneráveis, como pedestres, ciclistas e motociclistas. Ressalta-se que 93\% das mortes no trânsito ocorrem em países de baixa e média renda, com lesões fatais entre crianças e jovens de 5 a 29 anos. ${ }^{15}$

Diferentemente dos dados deste estudo, pesquisas elaboradas com base em serviços públicos de urgência e de emergência registraram percentuais inferiores a $7 \%$ dos atendimentos relacionados a distúrbios gastrointestinais. E, no que se refere aos distúrbios musculoesqueléticos, as taxas são inferiores a $2 \%$. Nesse sentido, levando-se em consideração que serviços públicos de urgências e de emergências registram altos índices de intervenções para distúrbios cerebrovasculares, entende-se que novos estudos precisam ser realizados, uma vez que os achados deste estudo distanciam-se dos padrões de ocorrência encontrados na literatura. ${ }^{1,5-6}$ Cabe ressaltar que o recorte analisado diz respeito a um serviço privado de PA e, desta forma, os achados podem ser influenciados pelos costumes e tradições da região onde o serviço está inserido.

Os dados indicam uma uniformidade na distribuição dos casos pelos meses do ano. No que refere aos dias da semana, verificou-se que o início da semana (terças e segundas-feiras) apresentou as maiores taxas de procura pelo serviço, enquanto o turno da manhã abrigou as maiores demandas.

Essa característica de acessibilidade ao serviço, levando em consideração os dias da semana e o horário, pode estar relacionada à baixa complexidade dos casos, permitindo aos usuários aguardarem o início da semana para receberem atendimento. Outro fator, potencialmente, influente desses casos diz respeito às altas cargas de trabalho, sem condições e 
equipamentos adequados. Essa prática torna muitos ambientes laborais estressantes, repletos de tarefas e de cobranças, levando à exaustão física e mental do colaborador, contribuindo para o adoecimento e suas manifestações clínicas durante o expediente de trabalho. Acredita-se, também, que, nesses dias, as pessoas estão em plena atividade, ou seja, mais vulneráveis à violência, aos acidentes de trânsito e a assaltos. ${ }^{16-18}$

O fluxo dos atendimentos em unidades de urgência e emergência é intenso, devido, também, à assistência prestada a usuários com doenças ou agravos de menor complexidade, que poderiam sofrer intervenção nos serviços de Atenção Básica de Saúde (ABS). ${ }^{19}$ A falta de conscientização dos usuários, em relação à complexidade do serviço, está evidenciada na elevada taxa de atendimentos classificados como pouco urgente (verde) e no tempo de permanência na unidade, com taxa próxima de $89 \%$ dos usuários recebendo alta no mesmo dia. São diversas as justificativas que levam o usuário ao PA. Dentre elas, a mais evidente, refere-se à alta densidade tecnológica, uma vez que exames podem ser realizados no momento do atendimento. Assim, torna-se possível fechar diagnóstico e iniciar tratamento instantâneo. ${ }^{20}$

A procura por suporte em instituições privadas deve-se a vários motivos, entre eles a falta de infraestrutura associada à falta de insumos e recursos humanos das unidades básicas de saúde. Estes fatores podem incentivar a população a adquirirem convênios e utilizarem serviços privados na expectativa de resolutividade das suas necessidades. A literatura menciona que pessoas com maior poder aquisitivo e maior escolaridade utilizam com maior frequência os serviços privados de saúde. O acúmulo de conhecimento, relacionado à escolaridade, sugestiona uma maior capacidade de interpretação dos agravos de saúde e suas consequências a curto e a longo prazo. ${ }^{19-20}$

Além do manejo adequado da equipe de saúde para o problema enfrentado pelo paciente, evidencia-se a necessidade de equalização das condições ambientais (moradia e saneamento básico), adesão a medidas sanitaristas como a vacinação, aleitamento materno e medidas 
Perfil epidemiológico dos atendimentos de um pronto atendimento privado do sul do Brasil| 14

promocionais e preventivas em saúde. ${ }^{21}$ Diferentemente dessa perspectiva, a identificação de quadros como traumatismo crânio encefálico, alterações neurológicas, cardiovasculares, intoxicações exógenas, parada cardiorrespiratórias, entre outros, sinaliza-nos a importância de contar como uma unidade de portas abertas. O atendimento imediato dos casos de urgência e de emergência, com infraestrutura adequada e equipe qualificada para o manejo do paciente, é fundamental para sustentação e organização do sistema de saúde. ${ }^{22}$

O elevado número de exames laboratoriais e radiológicos utilizados no serviço estudado agrega evidências no que se refere à baixa complexidade dos casos atendidos, tendo em vista a disponibilidade de outras ferramentas de diagnósticos. Entende-se que estes exames facilitam o diagnóstico rápido e preciso, principalmente em casos de trauma e acometimentos cardiorrespiratórios. Compreende-se, também, que, na prática médica, é imperativa a solicitação de exames destas naturezas pela facilidade de requerimento, execução e o desejo da certeza do diagnóstico. No entanto, essa prática contribui para gastos desnecessários. O Reino Unido, Europa e Itália são exemplos de modificação desse padrão terapêutico, pois, para enfrentar essa problemática, reorganizaram o atendimento investindo no diálogo com o paciente, anamnese completa, exame físico adequado e atualizações dos profissionais. Práticas revitalizadas que geraram uma maior precisão na avaliação clínica e, consequentemente, redução de custos. ${ }^{23-24}$

No período investigado, a maioria dos usuários atendidos recebeu alta após o tratamento. Nesse sentido, entende-se que a ABS tem papel definidor na construção e orientação da demanda para as demais esferas de saúde. Sua capacidade de intervenção pode influenciar, diretamente, a prevenção e a não cronicidade de doenças e/ou agravos constituintes das demandas atendidas nos níveis secundário e terciário do sistema de saúde. ${ }^{19}$

Dessa forma, acredita-se que a ABS pode influenciar usuários e coletividades no entendimento da utilização do sistema de saúde. Compreende-se, também, que é preciso levar em consideração a característica do serviço, público ou privado, os níveis de complexidade e as 
portas de acesso ao SUS. Cabe ressaltar que práticas preventivas e promocionais de saúde podem influenciar na reordenação de fluxo aos serviços, redução nos índices de adoecimento e/ou agravos a saúde..$^{7,16}$

Entende-se que a atenção secundária tem como objetivo prestar assistência resolutiva e qualificada aos casos agudos ou agudizados de natureza clínica, prestando os primeiros atendimentos aos casos cirúrgicos e/ou traumáticos, estabilizando-os. Além disso, realizar investigação diagnóstica inicial e, quando necessário, direcionar o encaminhamento para serviços hospitalares de maior complexidade. ${ }^{7,18}$

No entanto, agregam-se as discussões já realizadas que os atendimentos de maior complexidade, realizados pelo serviço em questão, ocorreram entre terça e segunda-feira e foram destinados a usuários adultos, economicamente ativos, e, consequentemente, mais expostos a fatores de risco. Pesquisadores e estudiosos ressaltam a importância de avaliações mais acuradas e atentas às populações vulneráveis definidas localmente. No que se refere à complexidade dos casos, estudos internacionais indicam decréscimo no número de atendimentos ao longo da semana, com aumento na segunda-feira, e menor procura aos finais de semana. Essa tendência demonstra que muitos usuários buscam o serviço sem estar apresentando uma emergência. ${ }^{25-26}$

A classificação de risco para os casos classificados na cor azul apresenta relação estatística com o tempo de permanência dos usuários no serviço. Nesse sentido, acredita-se que a baixa complexidade dos casos justifique esse dado, uma vez que 99,1\% dos atendimentos dizem respeito à soma das altas no mesmo dia e dos usuários que permaneceram no serviço por até um dia.

Segundo um estudo americano, que investigou as razões de procura dos usuários por serviços de emergência, entre as principais causas estão a dificuldade em conseguir ou o desconhecimento sobre como obter uma consulta ambulatorial, a crença de que seu problema de 
Perfil epidemiológico dos atendimentos de um pronto atendimento privado do sul do Brasil| 16

saúde não poderia esperar e o entendimento de que os serviços de emergência prestam assistência de melhor qualidade. ${ }^{27}$ Nessa perspectiva, uma pesquisa baseada nas prioridades de classificação de risco de um serviço público de atendimento apresenta semelhanças com o perfil aqui descrito. Segundo os autores, a utilização dos serviços de emergências pela população não reflete emergências. Entende-se que elas estejam ligadas à compreensão de utilização desses serviços, como uma porta qualificada de acesso ao sistema de saúde, desconsiderando a ABS, nicho destinado a absorver a demanda de baixa complexidade. ${ }^{28}$

\section{Conclusão}

Os resultados indicam diferenças geracionais e de gênero na utilização do serviço e evidencia o comportamento sazonal de utilização entre os dias da semana. As segundas-feiras, as terças-feiras e o turno da manhã apresentaram as maiores taxas de procura pelo serviço. A alta demanda de usuários com distúrbios gastrointestinais é uma característica que precisa ser explorada em outras pesquisas, tendo em vista seu distanciamento dos relatos encontrados na literatura.

A baixa complexidade dos atendimentos realizados e a procura ansiosa dos usuários em resolver suas necessidades, mesmo que essas não sejam caracterizadas como urgentes/emergentes, caracterizam, no que se refere à gravidade dos casos, o perfil da demanda atendida. Esse cenário pode encontrar explicação na garantia de acolhimento, por se tratar de um "serviço de portas abertas", e pela sua capacidade terapêutica e diagnóstica, o que impulsiona a ampla procura independente da complexidade clínica.

Os PAs são estruturas fundamentais na esfera da saúde e na sua organização, uma vez que podem influenciar na redução dos agravos a saúde, afetando as taxas de morbimortalidade e sequelas incapacitantes. Ao identificar o perfil epidemiológico da população atendida, é possível 
planejar ações, direcionar fluxo, capacitar profissionais, e colaborar com a formulação de políticas públicas de saúde.

Cabe ressaltar que, longe de esgotar os diferentes aspectos que compõem a temática dos atendimentos de urgência e emergência, este estudo retrata o perfil da demanda em uma unidade de PA privada, com fluxo composto por um recorte espacial específico e análise contida para o ano de 2017. Os recortes metodológicos aqui utilizados oportunizam o conhecimento das dinâmicas de atuação desse tipo de serviço, mas inviabilizam generalizações e requerem cuidado analítico ao realizar comparações com serviços públicos de urgência e emergência.

Os resultados apontam, em uma perspectiva ampliada, para a necessidade de incremento da ABS, visando à elaboração de estratégias capazes de impactar, de forma resolutiva, os problemas de saúde nessa esfera de atendimento. Com isso, entende-se que a soma de esforços governamentais, sociedade civil e trabalhadores de saúde pode melhorar o entendimento social, elaborando práticas e condutas capazes de influenciar a utilização dos serviços de PA. Nesse sentido, as políticas públicas de saúde podem agregar maiores esforços, no que se refere à educação dos usuários, com objetivo de aprimorar a compreensão do fluxo de atendimento dentro do sistema de saúde. Acredita-se que ações sistemáticas, nesse sentido, podem valorizar, socialmente, a ABS, além de promover melhores condições de vida e cidadania à população.

Por fim, ao estudar o perfil dos usuários atendidos por serviços de saúde privados, esta pesquisa corrobora com o delineamento do processo saúde-doença da população, contribuindo para a elaboração de políticas de saúde. A construção dessas políticas deve ser baseada em evidências locais, segundo as necessidades de cada coletividade, aumentando, assim, o sucesso da gestão e a qualidade da assistência nos diferentes níveis de atenção à saúde.

\section{Referências}

1. Frango BCTM, Batista REA, Campanharo CRV, Okuno MFP, Lopes MCBT. Associação do perfil de 
Perfil epidemiológico dos atendimentos de um pronto atendimento privado do sul do Brasil| 18

usuários frequentes com as características de utilização de um serviço de emergência. REME Rev Min Enferm. 2018;22:e-1071. doi: http://www.dx.doi.org/10.5935/1415-2762.20180001

2. Barreto RF, Gomes CZL, Silva RM, Signorelli AAF, Oliveira LF, Cavellani, et al. Pain and epidemiologic evaluation of patients seen by the first aid unit of a teaching hospital. Rev Dor. 2012;13(3):213-9. doi: https://doi.org/10.1590/S1806-00132012000300004

3. Organização Panamericana de Saúde (OPAS Brasil). 10 principais causas de morte no mundo [Internet]. 2018 [acesso em 2020 abr 29]. Disponível em: https://www.paho.org/bra/index.php?option=com_content\&view=article\&id=5638:10-principais-causasde-morte-no-mundo\&Itemid $=0$

4. Ministério da Saúde (BR), Departamento de Informática do SUS (DATASUS). Sistema de informações sobre mortalidade [Internet]. 2020 [acesso em 2020 abr 27]. Disponível em: http://www2.datasus.gov.br/DATASUS/index.php?area=02

5. Hora RS, Paiva EF, Sampaio ES, Oliveira JA, Souza VRS, Brandão PC. Caracterização do atendimento do serviço de atendimento móvel de urgência (Samu) às emergências clínicas. REME Rev Min Enferm. 2019;23:e-1256. doi: 10.5935/1415-2762.20190104

6. Rodriguez GCB, Dantas RAN, Dantas DV, Lima KRB, Lima MSM, Sarmento SDG, et al. Caracterização das vítimas de emergências clínicas atendidas por um serviço de atendimento préhospitalar móvel de urgência. Rev Nurs [Internet]. 2018 [acesso em 2020 abr 29];21(240):2173-7. Disponível em: http://www.revistanursing.com.br/revistas/240-Maio2018/atendimento_movel.pdf

7. Oliveira APC, Dal Poz MR, Craveiro I, Gabriel M, Dussault G. Fatores que influenciaram o processo de formulação de políticas de recursos humanos em saúde no Brasil e em Portugal: estudo de caso múltiplo. Cad Saúde Pública. 2018;34(2):0022416. doi: https://doi.org/10.1590/0102-311x00220416

8. Soares TCS, Marta CB, Silva RCL, Peregrino AAF, Santiago LC, Schutz V. Perfil dos usuários atendidos na sala vermelha de uma unidade de pronto atendimento $24 \mathrm{~h}$. Rev Enferm UFPE On Line [Internet]. 2016 [cited 2020 Apr 29];10(12):4619-27. Available from: https://periodicos.ufpe.br/revistas/revistaenfermagem/article/view/11531

9. Instituto Brasileiro de Geografia e Estatística (IBGE). IBGE Cidades [Internet]; 2018 [acesso em 2018 out 20]. Disponível em: https://cidades.ibge.gov.br/brasil/rs/taquara/panorama

10. Ministério da Saúde (BR), Secretaria de Atenção à Saúde, Política Nacional de Humanização da Atenção e Gestão do SUS. Acolhimento e classificação de risco nos serviços de urgência [Internet]. 2009 [acesso em 2020 maio 20]. Disponível em: http://bvsms.saude.gov.br/bvs/publicacoes/acolhimento_classificaao_risco_servico_urgencia.pdf

11. Santos RO, Ferreira LS, Carvalho FLO, Soares APG, Pereira RSF. Fatores que influenciam a baixa adesão masculina ao atendimento prestado pela estratégia de saúde da família sede II do município de 
Sítio do Quinto/BA. Rev Saúde UniAGES [Internet]. 2016 [acesso em 2020 abr 29];1(1):58-87. Disponível em: http://npu.faculdadeages.com.br/index.php/revistadesaude/article/view/4

12. Bega AG, Peruzzo HE, Lopes APAT, Dutra AC, Decesaro MN, Marcon SS. A busca de assistência à saúde em serviços de pronto-atendimento por mulheres adultas. Rev Pesq Cuid Fundam [Internet]. 2017 $\begin{array}{llll}\text { [cited } 2020 & \text { Apr } & \text { 29];9(1):1-14. Available }\end{array}$ from: http://www.seer.unirio.br/index.php/cuidadofundamental/article/view/5395

13. Melo MAS, Coleta MFD, Coleta JAD, Bezerra JCB, Castro AM, Melo ALS, et al. Percepção dos profissionais de saúde sobre os fatores associados à subnotificação no Sistema Nacional de Agravos de Notificação (Sinam). Rev Adm Saúde. 2018;18(71). doi: http://dx.doi.org/10.23973/ras.71.104

14. Krug EG, Dahlberg LL, Mercy JA, Zwi AB, Lozano R. World report on violence and health [Internet]. Geneva: World Health Organization; 2002 [cited 2020 Apr 29]. Available from: https://www.who.int/violence_injury_prevention/violence/world_report/en/

15. Organização Panamericana de Saúde (OPAS Brasil). Folha informativa - Acidentes de trânsito [Internet]. 2019 [acesso em 2020 abr 27]. Disponível em: https://www.paho.org/bra/index.php?option=com_content\&view=article\&id=5147:acidentes-de-transitofolha-informativa\&Itemid=779

16. Reibnitz Júnior C, Freitas FL, Ramos FRS, Tognoli H, Amante LN, Cutolo LRA, et al. Atenção integrada à saúde do adulto: medicina. $2^{\underline{a}}$ ed. Florianópolis: Universidade Federal de Santa Catarina; 2016.

17. Soldera LLO, Martins LG. Síndrome de burnout: conceitos e observações para gestores de recursos humanos. Leopoldianum [Internet]. 2017 [acesso em 2020 abr 29];43(119-20):143-53. Disponível em: http://periodicos.unisantos.br/leopoldianum/article/view/741/623

18. Holanda FL, Marra CC, Cunha ICKO. Professional competency profile of nurses working in emergency services. Acta Paul Enferm. 2015;28(4). doi: https://doi.org/10.1590/1982-0194201500053

19. Acosta AM, Lima MADS. Usuários frequentes de serviços de emergência: fatores associados e motivos de busca por atendimento. Rev Latinoam Enferm. 2015;23(2):337-44. doi: https://doi.org/10.1590/0104-1169.0072.2560

20. Dilélio AS, Tomasi E, Thumé E, Silveira DS, Siqueira FCV, Piccini RX, et al. Padrões de utilização de atendimento médico-ambulatorial no Brasil entre usuários do Sistema Único de Saúde, da saúde suplementar e de serviços privados. Cad Saúde Pública. 2014;30(12):2594-606. doi: http://dx.doi.org/10.1590/0102-311X00118713

21. Brandt KG, Antunes MMC, Silva GAP. Diarreia aguda: manejo baseado em evidências. J Pediatr (Rio J). 2015;91(6 Suppl 1):S36-43. doi: https://doi.org/10.1016/j.jped.2015.06.002. 
Perfil epidemiológico dos atendimentos de um pronto atendimento privado do sul do Brasil| 20

22. Morton PG, Fontaine DK. Cuidados críticos de enfermagem. 9aㅗ ed. Rio de Janeiro: Guanabara Koogan; 2013.

23. Rocha ECB, Lima LJL, Almeida MVG, Lopes MR. Necessidade de gerenciamento dos gastos com exames laboratoriais no Brasil. Rev Educ Val São Francisco [Internet]. 2018 [acesso em 2020 abr 29];8(15):112-28. Disponível em: http://www.periodicos.univasf.edu.br/index.php/revasf/article/view/178

24. Silva HR, Faleiro RD, Carlos MCF, Ietsugu MV, Fonseca PR. Demanda de exames radiográfico em serviços de urgência e emergência em Barra do Graças-MT. Tékhne Lógos [Internet]. 2018 [acesso em 2020 abr 29];9(1):99-105. Disponível em: http://revista.fatecbt.edu.br/index.php/tl/article/view/510/0

25. Hemmi APA, Conceição JA, Santos DDM. Representação sociais de homens sobre saúde e doença: contribuições para o cuidado. Rev Enferm Cent Oeste Min. 2015;5(1):1457-68. doi: http://dx.doi.org/10.19175/recom.v0i0.642

26. Zachariasse JM, Seiger N, Rood PPM, Alves CF, Freitas P, Smit FJ, et al. Validity of the Manchester Triage System in emergency care. PLoS One. 2017;12(2). doi: http://dx.doi.org/10.1371/journal.pone.0170811

27. Doran KM, Colucci AC, Wall SP, Williams ND, Hessler RA, Goldfrank LR. Reasons for emergency department use: do frequent users differ. Am J Manag Care. [Internet]. 2014 [cited 2020 Apr 29];20(11):506-14. Available from: http://www.ajmc.com/journals/issue/2014/2014-vol20-n11/Reasons-forEmergency-Department-Use-Do-Frequent-Users-Differ/

28. Marconato RS, Monteiro MI. Risk classification priorities in an emergency unit and outcomes of the service provided. Rev Latinoam Enferm. 2017;25. doi: http://dx.doi.org/10.1590/1518-8345.2345.2974

\section{Autor correspondente}

André Luis Machado Bueno

E-mail: almachadobueno@gmail.com

Endreço: Av. Dr. Carlos Barbosa, 530 - Apto 202. Porto Alegre, RS, Brasil.

CEP: $90880-000$

\section{Contribuições de Autoria}

1 - Raíssa Hehn

Concepção e planejamento do projeto de pesquisa, coleta, análise dos dados e redação do relatório de pesquisa.

\section{2 - André Luis Machado Bueno}

Oorientação na construção do projeto e revisão crítica da versão final do relatório de pesquisa.

\section{Como citar este artigo}

Hehn R, Bueno ALM. Perfil epidemiológico dos atendimentos de um pronto atendimento privado do sul do Brasil. Rev. Enferm. UFSM. 2020 [Acesso em: Anos Mês Dia]; vol.10 e58: 1-20. DOI:https://doi.org/10.5902/2179769237989 